\title{
Development And Validation of An Eco-Friendly and High-Throughput HPLC Method To Quantify Pyrethroid and Pyriproxyfen in Long-Lasting Insecticide-Treated Nets
}

\section{Kyle J. Walker}

Vector Biology Department, Liverpool School of Tropical Medicine, Pembroke PI, Liverpool L3 5QA Christopher T. Williams

Vector Biology Department, Liverpool School of Tropical Medicine, Pembroke PI, Liverpool L3 5QA Folasade 0. Oladepo

Vector Biology Department, Liverpool School of Tropical Medicine, Pembroke PI, Liverpool L3 5QA John Richard Lucas

Technical Consultant, Cowleigh Park Farm, Cowleigh Road, Malvern WR13 5HJ

\section{David Malone}

Innovative Vector Control Consortium, Liverpool School of Tropical Medicine, Pembroke Place, Liverpool L3 5QA

\section{Mark J.I. Paine}

Vector Biology Department, Liverpool School of Tropical Medicine, Pembroke PI, Liverpool L3 5QA

Hanafy M. Ismail ( $\square$ hanafy.ismail@lstmed.ac.uk)

Vector Biology Department, Liverpool School of Tropical Medicine, Pembroke PI, Liverpool L3 5QA

\section{Research Article}

Keywords: HPLC, High-throughput analysis, Pyrethroid and pyriproxyfen insecticides, Sustainable solvent use, LLINs, Malaria, Vector Control

Posted Date: December 29th, 2021

DOI: https://doi.org/10.21203/rs.3.rs-1197404/v1

License: (c) (1) This work is licensed under a Creative Commons Attribution 4.0 International License. Read Full License 


\section{Abstract}

Long-lasting insecticide-treated nets (LLINs) are essential to preventing malaria transmission. The LLINs should last for at least three years, even after repeated washings. Currently, tracking insecticides in LLINs is cumbersome, costly, and requires specialized equipment and hazardous solvents. We therefore developed a low-resource, high-throughput method for detecting insecticides in LLINs. In order to extract insecticides from polyethylene, LLIN samples were heated for 45 minutes at $85^{\circ} \mathrm{C}$ in 1-propanol-heptane containing dicyclohexylphthalate as an internal standard. Sample size was reduced to $\sim 0.2 \mathrm{~g}$ for reduced extraction volume, which is $90 \%$ less than what was recommended. We optimized HPLC chromatographic conditions to assess pyrethroid and pyriproxyfen content in polyethylene-based LLINs. The method is capable of quantifying levels $\geq 0.0015 \%$ permethrin, $0.00045 \%$ alpha-cypermethrin and $0.00025 \%$ pyriproxyfen $(\mathrm{w} / \mathrm{w})$ in polyethylene, allowing tracking the insecticides before and after LLINs use. A variety of LLINs can be evaluated with this method, including those with $1 \%$ pyriproxyfen (pyriproxyfenLLIN) or $2 \%$ permethrin (Olyset ${ }^{\circledR}$ Net), $1 \%$ pyriproxyfen and $2 \%$ permethrin (Olyset ${ }^{\circledR}$ Duo), or $0.55 \%$ pyriproxyfen combined with $0.55 \%$ alpha-cypermethrin (Royal Gaurd ${ }^{\circledR}$ ). Analysis of 120 samples (40 nets) per run can be done with high precision and accuracy, reducing labour time and costs whilst reducing the environmental impact of organic solvents.

\section{Introduction}

Human deaths due to malaria declined by approximately $50 \%$ between 2000 and $2015^{1,2}$, primarily due to the development, scale-up and universal distribution of long-lasting insecticide-treated nets (LLINs), the preferred form of insecticide-treated nets (ITNs) ${ }^{1}$. Nearly 2.2 billion ITNs have been delivered worldwide since 2004, of which 1.9 billion (86\%) were supplied to Sub-Saharan Africa ${ }^{3}$ preventing up to $68 \%$ of the malaria cases in the region ${ }^{2}$. ITNs reduce malaria transmission by acting as a physical barrier to block mosquito-human contact and killing and repelling mosquitoes by the insecticide ${ }^{3,4}$.

Pyrethroid insecticides such as permethrin and alpha cypermethrin (Fig. 1) are neurotoxic voltage-gated sodium channel blockers. The World Health Organization (WHO) recommends using pyrethroid in ITNs as they are highly toxic to mosquitoes but not to mammals ${ }^{3}$. They also have a less irritating smell to humans and excellent excito-repellency efficacy, the characteristic responsible for stimulating behavioural avoidance responses of mosquito malaria vectors from the pyrethroid-treated surface and preventing blood-feeding ${ }^{3,4}$. However, since 2016, there have been worrying signs of malaria resurgence in many areas of Sub-Saharan Africa, primarily due to the rapid evolution of pyrethroid resistance in mosquitoes ${ }^{3}$.

With compelling evidence for the impact of pyrethroid resistance on malaria control ${ }^{5,6}$, LLINs that incorporate two insecticides with different modes of action are being introduced ${ }^{7,8}$. Combining two insecticides with varying modes of action can delay the development of resistance and extend the lifetime of both active ingredients ${ }^{7,9}$. The Olyset ${ }^{\circledR}$ Duo (Sumitomo Chemical Co. Ltd.), for instance, is a prototype net that contains the pyrethroid permethrin plus pyriproxyfen (Fig. 1). The latter is a juvenile 
growth hormone analogue that prevents metamorphosis in larvae and can reduce the fecundity and longevity of adult mosquitoes. Olyset ${ }^{\circledR}$ Duo has been demonstrated to kill pyrethroid-resistant Anopheles gambiae mosquitoes and sterilize surviving blood-fed mosquitoes ${ }^{7,10,11}$. Recently, Disease Control Technologies (USA) has introduced Royal Guard®, a new type of pyrethroid-pyriproxyfen based LLIN that contains a mixture of alpha-cypermethrin and pyriproxyfen incorporated into the monofilament yarn of a polyethylene polymer. Royal Guard® Net was prequalified by WHO in March 2019 and can improve malaria vector control as indicated by its increased efficacy against $A n$. gambiae sl mosquitoes before and after 20 standardized washes in laboratory and experimental hut studies ${ }^{12}$.

New ITN products must adhere to guidelines from the WHO Prequalification Team for Vector Control Products (PQT-VC) in relation to insecticide content, wash resistance, storage stability, bio-efficacy, and field trials ${ }^{13}$. This requires the parallel development of analytical approaches for new product quality control assessment (QCA). Also, given the imminent arrival of new LLINs into the ITN market, the development of 'accessible' methods for quantifying insecticides will be necessary for stakeholders such as procurement agencies and vector control operatives to monitor the quality of the bed nets being used for malaria control operations. Collaborative International Pesticides Analytical Council (CIPAC) methods that utilize chromatographic techniques are available for insecticide quantification ${ }^{14,15}$ and referenced in WHO testing specifications for LLINs ${ }^{13}$. For instance, the standard CIPAC protocol for analyzing pyriproxyfen content in LLIN (715/LN/M, CIPAC Handbook 0, page 143) is suitable for determining pyriproxyfen content in nets containing pyriproxyfen as the only active ingredient and in mixtures with permethrin ${ }^{15}$. Also, the HPLC method for pyrethroid quantification has been developed to provide a universal protocol for detecting and analyzing pyrethroids from both coated and incorporated nets ${ }^{16}$. However, all available methods rely on a large sample size ( 2 grams of net mass equivalent to $\sim 400$ $\mathrm{cm}^{2}$ ), consume large volumes of organic solvents that require large extraction vessels and use a rotary evaporator for sample concentration (Fig. 2). Contrary to the aims of green chemistry, there are potential adverse effects to the environment resulting from large volume solvent consumption ${ }^{17}$. Furthermore, these methods are labour-intensive, time-consuming and costly, providing barriers to their being implemented in country for routine QCA.

Here we have modified the sampling method of LLINs to reduce the sample size of LLIN and the consumption of organic solvent to simplify the extraction and quantification procedure for insecticide(s) in LLINs. In addition, we have optimized the chromatographic conditions used in the standard CIPAC protocol for QCA of pyriproxyfen-LLIN ${ }^{15}$ to improve the HPLC sensitivity for pyrethroid quantification alone or in combination with pyriproxyfen. A range of prototype and commercial LLINs, i.e. PyriproxyfenNet (Pyriproxyfen), Olyset ${ }^{\circledR}$ Net (Permethrin), Olyset ${ }^{\circledR}$ Duo (permethrin and pyriproxyfen mixture) and Royal Guard $\circledast$ (alpha-cypermethrin and pyriproxyfen mixture) were used to assess the optimized method for insecticide(s) quantification specificity, accuracy, precession, and reproducibility. Results indicate that the new method is suitable for quantifying insecticide(s) content in LLINs containing pyriproxyfen and/or pyrethroid active ingredient. The new method provides high throughput analytical capacity for insecticide(s) quantification in LLINs. 


\section{Methods}

\section{Reagents}

Technical grade insecticide standards for HPLC analysis were obtained from Sigma Aldrich - permethrin $98.3 \%$ purity (57.8\% trans-isomer, $40.5 \%$ cis-isomer); alpha-cypermethrin, $\geq 98 \%$ purity). HPLC grade acetonitrile ( $\geq 99 \%)$, water and heptane were obtained from Fisher Chemicals. 1-propanol ( $\geq 99 \%)$ was obtained from Across Organics. Four types of LLIN were obtained from different suppliers (Table 1).

Table 1

Manufacturer and insecticide information for LLINs.

\begin{tabular}{|c|c|c|c|c|}
\hline LLIN Name & Manufacturer & Denier & Material & $\begin{array}{l}\text { Active ingredient } \\
\text { concentration }\end{array}$ \\
\hline $\begin{array}{l}\text { Pyriproxyfen- } \\
\text { Net }\end{array}$ & $\begin{array}{l}\text { Sumitomo Chemical } \\
\text { (Japan) }\end{array}$ & 150 & Polyethylene & Pyriproxyfen $(10 \mathrm{~g} / \mathrm{Kg})$ \\
\hline Olyset ${ }^{\circledR}$ Net & $\begin{array}{l}\text { Sumitomo Chemical } \\
\text { (Japan) }\end{array}$ & 150 & Polyethylene & Permethrin $(20 \mathrm{~g} / \mathrm{Kg})$ \\
\hline Olyset巴 Duo & $\begin{array}{l}\text { Sumitomo Chemical } \\
\text { (Japan) }\end{array}$ & 150 & Polyethylene & $\begin{array}{l}\text { Permethrin }(20 \mathrm{~g} / \mathrm{Kg})+ \\
\text { Pyriproxyfen }(10 \mathrm{~g} / \mathrm{Kg})\end{array}$ \\
\hline $\begin{array}{l}\text { Royal } \\
\text { Guard } \circledast\end{array}$ & $\begin{array}{l}\text { Disease Control } \\
\text { Technologies, LLC (USA) }\end{array}$ & 120 & Polyethylene & $\begin{array}{l}\text { Alpha-cypermethrin }(5.5 \\
\mathrm{g} / \mathrm{Kg})+ \text { Pyriproxyfen }(5.5 \\
\mathrm{g} / \mathrm{Kg})\end{array}$ \\
\hline
\end{tabular}

HPLC analysis was performed with a Dionex UltiMate 3000 comprising an autosampler (WPS $3000 \mathrm{SL}$ ), quaternary pump (LPG 3400 SD), and variable wavelength detector (VWP 3410 RS). Peak areas were obtained using Chromeleon software (Chromeleon 7.2 SR4). The column used was a Hypersil GOLD C18 column ( $75 \AA, 250 \times 4.6 \mathrm{~mm}, 5$ - $\mu \mathrm{m}$ particle size; Thermo Scientific). Peak purity analysis was carried out using a Thermo Fisher Scientific Vanquish Core HPLC System comprised of a Vanquish ${ }^{\text {TM }}$ Split Sampler (VC-A12-A), Vanquish ${ }^{\text {TM }}$ Column Compartment (VC-C10-A), Vanquish ${ }^{\text {TM }}$ Binary Pump (VC-P10-A), and Vanquish $^{\text {TM }}$ Diode Array Detector; multiple wavelength detector (VC-D11-A).

\section{Optimized test method summary}

The method below outlines a single analysis of a single net. The methods for the validation experiments are outlined in later experimental sections. Whole nets consisting of five panels were tested. A small square (approximately $25 \times 25 \mathrm{~cm}^{2}$ ) was cut from each to perform a representative analysis of the whole net. These are laid on top of each other, and a small disc $\left(\sim 8 \mathrm{~cm}^{2}\right)$ cut from each. The total weight of the five discs was recorded before transferring to a 10 glass tube. Five millilitres of the extraction solution (1propanol: heptane $(1: 9 \mathrm{v} / \mathrm{v}))$ containing $100 \mu \mathrm{g} /$ dicyclohexyl phthalate [DCP] as an internal extraction controlwas added, ensuring all the net were submerged in the solution. The glass tubes were capped with tin foil and a lid and heated at $85^{\circ} \mathrm{C}$ for 45 minutes using a Dri-Block ${ }^{\circledR}$ (Techne) heater. One milliliter was then transferred to a new glass tube and evaporated at $60^{\circ} \mathrm{C}$ under compressed air, then resuspended in 1 acetonitrile and vortexed for one minute at 2500-3000 rpm before decanting into a 1.5 microcentrifuge 
tube. The sample wasfiltered through a PTFE $0.2 \mu \mathrm{m}$ filter before transferring $100 \mu \mathrm{l}$ to an HPLC vial for analysis. Standards of concentrations $(31.25 \mu \mathrm{g} /, 62.5 \mu \mathrm{g} /, 125 \mu \mathrm{g} /, 250 \mu \mathrm{g} /, 500 \mu \mathrm{g} /)$ were prepared for each insecticide present in the nets being analysed. The HPLC method incorporated an isocratic mobile phase of $70 \%$ acetonitrile and $30 \%$ water, a $1 / \mathrm{min}$ flow rate, 40 -minute run time and an analysis wavelength of $226 \mathrm{~nm}$. The quantities of permethrin and pyriproxyfen in $\mathrm{g} / \mathrm{kg}$ are calculated from standard curves produced from the known standard concentrations and corrected against the internal DCP control ss. The final insecticide content in $\mathrm{g} / \mathrm{kg}$ was estimated using the following equation:

$$
I=\left(\frac{x}{a}\right) \times\left(\frac{0.001}{m}\right) \times C \times \mathrm{f}
$$

where: / is the insecticide content in $\mathrm{g} / \mathrm{kg}$, and $x$ is the insecticide peak area at $226 \mathrm{~nm}$, (for permethrin the cis-and trans-isomer peak areas were combined). $a$ is the slope of the relevant insecticide standard curve. $m$ is the mass of the net sample. $C$ is the internal standard correction factor, calculated by dividing the average peak area of DCP controls by the DCP peak area obtained for the sample. $f$ is the sample dilution factor .

\section{Specificity}

To check the method specificity, chromatogram peaks of extraction solutions from Olyset $\circledast$ Duo $\circledast$ and Royal Guard $\circledast$ were compared with that of analytical grade insecticides (permethrin and pyriproxyfen). We confirmed there was no overlap of the insecticide peaks with either the internal control DCP or contamainants peaks co-extracted from polyethylene matrix. The chromatograms produced from these samples were also analyzed for any obvious peak shouldering, tailing or crossover. The insecticide peak retention time was also compared to that of the injected standards, and the percentage retention time was calculated from the following formula:

$\% \mathrm{RT}=R T_{\text {sample }} / R T_{\text {standard }} \times 100$

\section{Linearity}

Linear regression analysis was used to validate the linearity of HPLC for quantification of five working standard solutions of permethrin, alpha-cypermethrin and pyriproxyfen. The standards used ranged from $31.25 \mu \mathrm{g} /-500 \mu \mathrm{g} /$ as produced during the net analysis. The average peak area, standard deviation, and relative standard deviation (RSD\%) were recorded for each insecticide concentration. By injecting $20 \mu \mathrm{l}$ of insecticide concentrations $31.25,62.5,125,250$ and $500 \mu \mathrm{g} /$, the response should be linear with $R^{2}>0.9$. The linearity was evaluated by generating the calibration curves presented by the following linear regression analysis equation:

$y=a x+b \ldots \ldots$ (eq. 1) 
The linearity was obtained by plotting the peak areas $(y, m A U)$ of insecticide versus injected standard concentration $(\mu \mathrm{g} /)$ onto a column and by the value of their correlation coefficients $\left(R^{2}\right)$. For each of the three standard curves produced, the slope value is recorded. The average slope (a), standard deviation ( $\sigma$ ) and \%RSD of these slopes are also reported.

\section{Limit of detection (LoD) and limit of quantification (LoQ)}

LoD and LoQ assays were performed for both insecticides. According to the HPLC conditions described above, a $20 \mu \mathrm{l}$ of standard curve ranging from $0.007-250 \mu \mathrm{g} /$ was injected in triplicate. The LoD and LoQ were calculated by regression analysis slope (a) obtained from "eq. 1" and the standard deviation ( $\sigma$ ) value of the line obtained by analyzing these low-concentration solutions and following equations:

$L o D=3.3 \sigma / a \ldots \ldots . .($ eq. 2$)$

$L O Q=10 \sigma / a \ldots \ldots . .($ eq. 3$)$

\section{Accuracy and precision}

A recovery experiment was conducted to confirm that insecticides content was determined accurately with high precision. The samples subjected to this assessment were untreated nets fortified with concentrations of permethrin and pyriproxyfen at the specification level for each insecticide. Four nets were analyzed per concentration. The results were analyzed, and the following equation was used for the recoveries of the insecticides calculations:

$$
R=\frac{C}{C S} \times 100
$$

Where R: recovery \%, C: observed concentration of the insecticide $(\mu \mathrm{g} /)$ and Cs: fortified concentration $(\mu \mathrm{g} /)$ permethrin.

\section{Heat stability}

A comparative assay was performed to assess the stability of the insecticides when heated to $85^{\circ} \mathrm{C}$ for 45 minutes, comparing results with and without heating. For the heat stability experiment, 5 of insecticide at two concentrations, 0.4 and $0.2 \mathrm{mg} /(\mathrm{w} / \mathrm{v})$ in extraction solution were heated in triplicate at $85^{\circ} \mathrm{C}$ for 45 minutes. 1 of the solution was removed, evaporated, and reconstituted in 1 of HPLC-grade acetonitrile for HPLC analysis. In parallel, 1 unheated samples from the insecticide standard were evaporated and reconstituted in 1 acetonitrile to compare HPLC chromatograms of heated versus unheated treatments. All samples were then treated the same as described in the test method. The average insecticide recovered, standard deviation and \%RSD for heating and non-heating methods were reported for each insecticide.

\section{Reproducibility test}


To assess the method reproducibility, 24 new nets from Olyset ${ }^{\circledR}$ and Olyset ${ }^{\circledR}$ Duo (Table 1 ) were analyzed in triplicate. For Royal Guard $\circledast$ LLIN, one net was analyzed ten times alongside 30 net analyzed in a single replicate. All net samples were extracted and analyzed following the modification described above.

\section{Results}

\section{Improvement of HPLC analysis}

To increase the HPLC sensitivity for the simultaneous analysis of pyriproxyfen and pyrethroids in LLINs, we optimized the analytical chromatographic conditions in the standard CIPAC protocol recommended for quantifying pyriproxyfen in pyriproxyfen-LLIN ${ }^{15}$. Olyset ${ }^{\circledR}$ Duo LLIN manufactured with $20 \mathrm{~g} / \mathrm{kg}$ permethrin $(2 \% \mathrm{w} / \mathrm{w})$ and $10 \mathrm{~g} / \mathrm{kg}$ pyriproxyfen $(1 \% \mathrm{w} / \mathrm{w})$ and Royal Guard $\circledast$ LLIN manufactured with 5.5 $\mathrm{g} / \mathrm{kg}$ alpha-cypermethrin $(0.55 \%)$ and $5.5 \mathrm{~g} / \mathrm{kg}$ pyriproxyfen $(0.55 \%)$ were used as the test materials for HPLC method improvement. Extracts from $\sim 0.2 \mathrm{~g}$ of LLIN were investigated for detection sensitivity using a Vanquish ${ }^{\mathrm{TM}}$ Diode Array Detector (VC-D11-A) at shorter wavelengths of 226 and $232 \mathrm{~nm}$ compared to the recommended wavelength of $254 \mathrm{~nm}^{15}$. The resulting chromatograms are presented in Fig. 3 . All three insecticides produced the highest peak heights and corresponding peak areas at $226 \mathrm{~nm}$ (Fig. 3). At this wavelength, the greatest sensitivity was recorded for pyriproxyfen with LoD and LoQ of $0.04 \mu \mathrm{g} /(1$ $\mathrm{mg} / \mathrm{kg}$ net) and $0.1 \mu \mathrm{g} /(2.5 \mathrm{mg} / \mathrm{kg}$ net) respectively, followed by alpha-cypermethrin with LoD and LoQ of $0.06 \mu \mathrm{g} /(1.5 \mathrm{mg} / \mathrm{kg})$ and $0.18 \mu \mathrm{g} /(4.5 \mathrm{mg} / \mathrm{kg})$ respectively, and permethrin (cis and trans)) with LoD and LoQ of $2 \mu \mathrm{g} /(5 \mathrm{mg} / \mathrm{kg}$ net) and $0.6 \mu \mathrm{g} /(15 \mathrm{mg} / \mathrm{kg}$ net), respectively. DCP with a retention time well separated from the target insecticides was used as an internal standard to correct for volume errors and to ensure high reproducibility between samples. Four well-separated peaks of pyriproxyfen, DCP, transpermethrin and cis-permethrin were obtained with Olyset ${ }^{\circledR}$ Duo sample (Fig. 3A), and three separat peaks, pyriproxyfen, DCP and alpha-cypermethrin were obtained with Royal Guard® sample (Fig. 3B). An ambient column temperature $\left(23^{\circ} \mathrm{C}\right)$ was also used to ensure the method suitability across different laboratory settings. At this temperature, the optimized acetonitrile/water mobile phase ratio $70: 30(\mathrm{v} / \mathrm{v})$, which was slightly higher than the 66.6-33.3 (v/v) recommended method (CIPAC), produced symmetric analyte peaks with no sign of peak abnormalities and clear analyte separation (Fig. 3). Under these conditions the run times for Olyset ${ }^{\circledR}$ Duo and Royal Guard $\circledast$ were 40 min (Fig. 3A) and 30 min (Fig. 3B) respectively compared with 60 min per run in the standard CIPAC method ${ }^{15}$.

\section{Specificity}

The improved method was also assessed for method sepecificity to test its ability to measure accurately and specifically the insecticide of interest in the presence of other components that may be coextracted from the net matrix. Therfore, insecticide peaks determined in both samples were further investigated for the presence of visible interferences (shoulders) by comparison with retention times from insecticide standard injections. Sample retention time of analytes matched the standards with calculated percentage retention times of $100.11 \%$ (pyriproxyfen), $100.1 \%$ (DCP), $100.23 \%$ (trans-permethrin), $100.22 \%$ (cispermethrin) for sample extracted from Olyset ${ }^{\circledR}$ Duo (Fig. S1). Similarly, samples extracted from Royal 
Guard® Net exhibited $100.11 \%$ and $100.07 \%$ matching retention time for pyriproxyfen and alphacypermethrin, respectively (Fig. S2). In addition, the average peak purities for pyriproxyfen (997), transpermethrin (1000) and cis-permethrin (1000) from sample solutions extracted from Olyset巴 Duo Net matched the pure analyte peak factor of 1000 (Fig. S1) and for pyriproxyfen (998) and alphacypermethrin (1000) extracted from Royal Guard® Net (Fig. S2).

\section{Linearity}

The linearity of the method was examined using a concentration range that encompassed $8-125 \%$ of the target sample concentration for pyriproxyfen, $4 \%-120 \%$ for permethrin and $16-110 \%$ for alphacypermethrin. As presented in Table 2, a linear relationship was obtained between peak area and total concentration of permethrin, alpha-cypermethrin and pyriproxyfen with regression coefficient values close to $1.0\left(R^{2}>0.9994\right)$. For all tested insecticides, the $Y$ intercepts were effectively zero. The slope agreement was $\leq 5 . \%$ relative standard deviation (\% RSD) for permethrin, $\leq 2.2 \%$ for alpha-cypermethrin and $\leq$ $0.28 \%$ for pyriproxyfen.

Table 2

Linearity parameters, Regression Equations, Correlation Coefficients $\left(\mathrm{R}^{2}\right)$, and Standard Deviations (SD) Found During Linearity, LoQ, and LoD Testing*.

\begin{tabular}{|c|c|c|c|c|c|}
\hline Insecticide & Amount interval & Equation & $\mathbf{R}^{2}$ & Slope \pm SD & \%RSD \\
\hline $\begin{array}{l}\text { Permethrin }{ }^{a} \\
(\text { trans }+c i s)\end{array}$ & $\begin{array}{l}31.25-1000 \mu \mathrm{g} /(0.625- \\
20 \mu \mathrm{g})\end{array}$ & $\begin{array}{l}Y= \\
1.0517 X+8.9\end{array}$ & 0.9996 & $\begin{array}{l}1.0517 \pm \\
0.007\end{array}$ & 0.66 \\
\hline $\begin{array}{l}\text { Permethrin }{ }^{\mathrm{b}} \text { (trans } \\
+ \text { cis) }\end{array}$ & $\begin{array}{l}0.24-250 \mu \mathrm{g} /(4.8 \mathrm{ng}-5 \\
\mathrm{ug})\end{array}$ & $\begin{array}{l}Y=0.9938 X- \\
0.4\end{array}$ & 0.9994 & $\begin{array}{l}0.9938 \pm \\
0.06\end{array}$ & 5.8 \\
\hline $\begin{array}{l}\text { Alpha- } \\
\text { cypermethrin }\end{array}$ & $\begin{array}{l}31.25-500 \mu \mathrm{g} /(0.625 \text { - } \\
10 \mu \mathrm{g})\end{array}$ & $\begin{array}{l}Y=1.0384 X- \\
5.8\end{array}$ & 0.9994 & $\begin{array}{l}1.0384 \pm \\
0.0004\end{array}$ & 0.04 \\
\hline $\begin{array}{l}\text { Alpha- } \\
\text { cypermethrin }\end{array}$ & $\begin{array}{l}0.244-250 \mu \mathrm{g} /(4.8 \mathrm{ng}- \\
5 \mathrm{ug})\end{array}$ & $\begin{array}{l}Y=1.056733 X+ \\
0.5\end{array}$ & 0.9996 & $\begin{array}{l}1.056 \pm \\
0.02\end{array}$ & 2.2 \\
\hline Pyriproxyfen ${ }^{a}$ & $\begin{array}{l}31.25-500 \mu \mathrm{g} /(0.625 \text { - } \\
10 \mu \mathrm{g})\end{array}$ & $\begin{array}{l}Y=1.087 X+ \\
3.3\end{array}$ & 0.9999 & $\begin{array}{l}1.087 \pm \\
0.003\end{array}$ & 0.28 \\
\hline Pyriproxyfen ${ }^{b}$ & $\begin{array}{l}0.03-500 \mu \mathrm{g} /(0.61 \mathrm{ng}- \\
10 \mathrm{ug})\end{array}$ & $\begin{array}{l}Y=1.114 X+ \\
0.2\end{array}$ & 0.9999 & $\begin{array}{l}1.114 \pm \\
0.0125\end{array}$ & 0.13 \\
\hline \multicolumn{6}{|c|}{$\begin{array}{l}\text { * Chromatographic conditions used: } 70 \% \text { acetonitrile: } 30 \% \text { water isocratic mobile phase, } 1 / \text { min flow } \\
\text { rate, } 40 \text {-minute run time and an analysis wavelength of } 226 \mathrm{~nm} \text {. The column used for analysis was a } \\
\text { Hypersil GOLD C18 column ( } 75 \AA \AA \text {, } 250 \times 4.6 \mathrm{~mm}, 5-\mu \mathrm{m} \text { particle size; Thermo Scientific). }{ }^{\text {a Data }} \text { Data } \\
\text { obtained from linearity validation where }{ }^{b} \text { data obtained from LoQ and LoD calculation. A triplicate } \\
\text { set of standards were prepared for each insecticide. SD; standard deviation and \% RSD; relative } \\
\text { standard deviation (SD/Mean* } 100) \text {. }\end{array}$} \\
\hline
\end{tabular}

Accuracy and precision 
The insecticide recoveries from blank nets fortified with known quantities of insecticide are presented in Table 3. Permethrin recovery ranged from $101-111 \%$, alpha-cypermethrin recovery ranged from 97.7 $99.4 \%$, while pyriproxyfen recovery ranged from $105-107 \%$. The $\%$ RSD was $0.8 \%$ for both pyriproxyfen and alpha-cypermethrin and 3.8 for permethrin. Thus, the insecticide recovery for all insecticides examined was close to actual values with high precision.

Table 3

Accuracy and precision test for blank net fortified with permethrin, alpha-cypermethrin and pyriproxyfen active ingredients.

\begin{tabular}{|lllllll|}
\hline Sample Rep. & \multicolumn{2}{l}{ [Permethrin] } & \multicolumn{2}{l}{ [Alpha-cypermethrin] } & \multicolumn{2}{l|}{ [Pyriproxyfen] } \\
\cline { 2 - 7 } & $\mathbf{( g / k g})$ & Recovery \% & $\mathbf{( g / k g})$ & Recovery \% & $\mathbf{( g / k g )}$ & Recovery \% \\
\hline $\mathbf{1}$ & 20.3 & 101.5 & 5.362499 & 98.1 & 10.6 & 105.7 \\
\hline $\mathbf{2}$ & 20.9 & 104.4 & 5.384918 & 97.9 & 10.7 & 107.1 \\
\hline $\mathbf{3}$ & 21.0 & 105.1 & 5.46651 & 99.4 & 10.7 & 107.4 \\
\hline $\mathbf{4}$ & 22.2 & 111.1 & 5.374063 & 97.7 & 10.6 & 106.0 \\
\hline Mean \pm SD & $21.1 \pm 0.8$ & $105.5 \pm 4.0$ & $5.4 \pm 0.04$ & $98.3 \pm 0.76$ & $10.7 \pm 0.1$ & $106.6 \pm 0.8$ \\
\hline SD; standard deviation and \% RSD; relative standard deviation (SD/Mean*100) & \\
\hline
\end{tabular}

\section{Heat stability}

Given the chiral properties of pyrethroids and pyriproxyfen (Fig. 1) and the known vulnerability of pyrethroids to degrade or isomerize upon exposure to light, heat, and solvents ${ }^{18,19}$, the three insecticides were assessed for their heat stability and resistance to isomerization during extraction. The stability data for permethrin, alpha-cypermethrin and pyriproxyfen before and after heating at $85^{\circ} \mathrm{C}$ for 45 minutes are presented in Table 4. The corresponding HPLC chromatograms are shown in Fig. S3, Fig. S4 and Fig. S5 for permethrin, alpha-cypermethrin and pyriproxyfen, respectively. The quantity of the heated standards (permethrin, alpha-cypermethrin and pyriproxyfen) was equal to the unheated standards (Table 4). None of the examined insecticides demonstrated any signs of degradation/isomerization under the conditions tested (Fig S3, Fig. S4 and Fig. S5). 
Table 4

Stability of permethrin and pyriproxyfen active ingredients heated at $85^{\circ} \mathrm{C}$ for 45 minutes.

\begin{tabular}{|c|c|c|c|c|c|}
\hline Treatment & Insecticide RT & & $\mathrm{n}$ & [Insecticide] mg/ \pm SD & \%RSD \\
\hline Permethrin & Trans & Cis & & & \\
\hline $0.2 \mathrm{mg} /$ (Heated) & 25.5 & 29.6 & 3 & $0.207 \pm 0.00016$ & 0.08 \\
\hline $0.2 \mathrm{mg} /$ (Unheated) & $25.46 \pm 0.06$ & 29.5 & 3 & $0.202 \pm 0.00002$ & 0.01 \\
\hline $0.4 \mathrm{mg} /$ (Heated) & 25.5 & $29.56 \pm 0.06$ & 3 & $0.405 \pm 0.00028$ & 0.06 \\
\hline $0.4 \mathrm{mg} /$ (Unheated) & $25.5 \pm 0.06$ & $29.63 \pm 0.06$ & 3 & $0.399 \pm 0.00032$ & 0.08 \\
\hline \multicolumn{6}{|l|}{ Alpha-cypermethrin } \\
\hline $0.2 \mathrm{mg} /$ (heated) & $21.63 \pm 0.03$ & & 3 & $0.19 \pm 4.2 \mathrm{E}-05$ & 0.04 \\
\hline $0.2 \mathrm{mg} /$ (Unheated) & $21.65 \pm 0.05$ & & 3 & $0.19 \pm 2.7 \mathrm{E}-05$ & 0.04 \\
\hline $0.4 \mathrm{mg} /$ (Heated) & $21.61 \pm 0.02$ & & 3 & $0.41 \pm 0.001$ & 0.8 \\
\hline $0.4 \mathrm{mg} /$ (Unheated) & $21.61 \pm 0.06$ & & 3 & $0.41 \pm 0.0003$ & 0.33 \\
\hline \multicolumn{6}{|l|}{ Pyriproxyfen } \\
\hline $0.2 \mathrm{mg} /$ (Heated) & $11.6 \pm 0.0$ & & 3 & $0.19 \pm 0.0002$ & 0.12 \\
\hline $0.2 \mathrm{mg} /$ (Unheated) & $11.63 \pm 0.05$ & & 3 & $0.19 \pm 0.0001$ & 0.04 \\
\hline $0.4 \mathrm{mg} /$ (Heated) & $11.6 \pm 0.0$ & & 3 & $0.40 \pm 0.0032$ & 0.8 \\
\hline $0.4 \mathrm{mg} /$ (Unheated) & $11.56 \pm 0.06$ & & 3 & $0.39 \pm 0.0013$ & 0.33 \\
\hline
\end{tabular}

\section{Analysis of the total active ingredient(s) content from polyethylene-based LLIN formulations}

A range of LLIN formulations (Table 1) were used to evaluate the optimized method as a QCA method for insecticide(s) incorporated into polyethylene-based LLIN formulations and to validate the method reproducibility.

\section{Analysis of LLINs that incorporate a single insecticide}

Firstly, to investigate the agreement between the optimized method and CIPAC protocol for the analysis of pyriproxyfen content, a prototype net produced by Sumitomo (Table 1) was analyzed by the optimized method and compared with the standard CIPAC protocol for QCA of pyriproxyfen content in LLIN ${ }^{15}$. Samples were analyzed in duplicate as recommended by the standard CIPAC protocol ${ }^{15}$ and in quadruplet by the new method to account for possible variability in insecticide quantities due to mosaic distribution of a.i. in net material. Graphs comparing data obtained from the two protocols are presented in Fig. 4. The CIPAC method detected 11.25 and $11.7 \mathrm{~g} / \mathrm{kg}$ for LLIN1 and 2 respectively versus 10.5 and 
$11.25 \mathrm{~g} / \mathrm{kg}$ for the optimized method, which matched the manufactuers target dose $10 \pm 2.5 \mathrm{~g} / \mathrm{Kg}$. There was no significant difference in the average amount of pyriproxyfen extracted from the two nets by either method ( $P$ values of 0.68 and 0.87 for LLIN1 and LLIN2 (Fig. 4A) with differences between the two methods close to zero (Fig. 4B).

Next, we assessed the utility of the optimised method to quantify permethrin in Olyset ${ }^{\circledR}$ net, a representative set of standard manufactured LLINs recommended by WHOPES (currently known as PQTVC) that are incorporated with permethrin at a target dose of $20 \mathrm{~g} / \mathrm{kg}$ permethrin $(2 \% \mathrm{w} / \mathrm{w})$. To estimate method roubstness and reproducibility for analysis of permethrin content a 24 Olyset $\circledast$ nets were analysed in triplicate. Consistent with WHOPES recommendations ${ }^{13}$, none of the 24 nets scored an average content that differed from that declared by the manufacturer by more than $\pm 25 \%$ (Fig. $5 \mathrm{~A}$ ). Additionally, the method presented a satisfactory level of robustness and reproducibility, as indicated from QCA data shown in Fig. 5B. Out of 24 nets, 23 scored values within +/- 2SD of the $18.9 \mathrm{~g} / \mathrm{kg}$ average while the $21.1 \mathrm{~g} / \mathrm{kg}$ outlier remains within the WHOPES recommended range $20 \pm 5 \mathrm{~g} / \mathrm{kg}$. The relative standard deviation (\%RSD) of permethrin content was $<10 \%$ for all 24 nets analyzed in triplicate (Table S1), demonstrating the high precession and reproducibility of the HPLC method for permethrin quantification.

\section{Analysis of LLINs that incorporate two active ingredients}

Twenty four new Olyset $\circledast$ Duo ( $2 \%$ permethrin and $1 \%$ pyriproxyfen) were investigated for the simultaneous measurement of pyriproxyfen and permethrin content in LLIN polyethylene polymer following the optimized protocol. None of the 24 nets scored an average dual insecticide content that differed from the amount declared by the manufacture by more than $\pm 25 \%$ (Fig. $6 \mathrm{~A})$. The method showed robust reproducibility, as indicated by QCA data (Fig. 6B). All nets scored values within $+/-2 S D$ of the average of $19.1 \pm 1.3 \mathrm{~g} / \mathrm{kg}$ for permethrin and $10.4 \pm 0.5 \mathrm{~g} / \mathrm{kg}$ for pyriproxyfen (Fig. 6B). An indicative of the high precision of the HPLC method, the relative standard deviation (\%RSD) of permethrin and pyriproxyfen content obtained from all samples analyzed in triplicate was less than $10 \%$ (Table S2).

To establish the broader applicability of the new method for next-generation LLINs that are commercially available for malaria control, thirty Royal Guard ${ }^{\circledR}$ Net containing a mixture of alpha-cypermethrin and pyriproxyfen were assessed for insecticide content. None of the 30-nets scored an insecticide content that differed from the declared manufacturer's $5.5 \mathrm{~g} / \mathrm{kg}$ concentration by more than $\pm 25 \%$ (Fig. 7). However, a slight increase in the alpha-cypermethrin content has been noted, giving a value of $6.03 \pm 0.33 \mathrm{~g} / \mathrm{kg}$ (Fig. 7B).

The manufactured loading of active ingredient contents was further investigated by taking a random net from the 30 nets and subjecting it to five cycles of insecticide extraction in triplicates. The majority of the active ingredients were extracted in the first run (Fig. 6S). Pyriproxyfen quantity recovered in the first round of the extraction was $5.4 \pm 0.46 \mathrm{~g} / \mathrm{kg}$ and alpha-cypermethrin quantity was $5.6 \pm 0.14 \mathrm{~g} / \mathrm{kg}$, which is approximately equivalent to the manufacturer's reference value for both insecticides $(5.5 \pm 1.375 \mathrm{~g} / \mathrm{kg})$ 
(Fig. 6S). Compared to the first run, a negligible amount of the two active ingredients were recovered in the subsequent four runs, amounting to a residual amount of 0.02 and $0.6 \mathrm{~g} / \mathrm{kg}$ of pyriproxyfen and alpha-cypermethrin likely carried over from the first run (Fig. 6S).

To test the method reproducibility, ten samples from one Royal Guard ${ }^{\circledR}$ net were analyzed using the optmised protocol. Again, the method scored values similar to the manufacturer's average, $5.75 \pm 0.16$ and $5.43 \pm 0.34 \mathrm{~g} / \mathrm{kg}$ of pyriproxyfen and alpha-cypermethrin respectively (Fig. 7S). Analysis of ten samples with the optmised method also represented high reproducibility with \%RSD $<6.2$ for both active ingredients.

\section{Discussion}

We have developed a simplified approach for sample preparation, extraction and insecticide quantification from LLINs made from polyethylene polymers that incorporate pyrethroid and pyriproxyfen insecticides. The standard CIPAC protocol for the QCA of pyriproxyfen net recommends heating large amounts of net material $(\sim 2 \mathrm{~g})$ with 50 of the solvent mixture at $85-90^{\circ} \mathrm{C}$ in duplicate, which results in the production of a significant amount of solvent waste that if scaled for multiple nets could be problematic for public health and the environment ${ }^{17,20,21}$. Solvent selection guideline has identified heptane as a problematic but not hazardous solvent ${ }^{17,21}$. By reducing the sample size to $\sim 0.2 \mathrm{~g}$ we were able to reduce the solvent used for extraction by 10 -fold, providing greener chemistry and sustainable solvent use in chemical processing, and eliminating the need for rotary evaporation that prevents the facile evaporation of multiple samples for high throughput analysis of multiple LLINs. Chromatographic conditions were also optimized for the separation and quantitation of pyriproxyfen, permethrin and alpha-cypermethrin. The U.V. detection wavelength of $226 \mathrm{~nm}$ and mobile phase composition of $70 \%$ acetonitrile in water has helped to achieve higher sensitivity for insecticide detection and quantification with the small sample size $(0.2 \mathrm{~g})$ at shorter $30-40 \mathrm{~min}$ run time relative to CIPAC $(60 \mathrm{~min})^{15}$.

The extraction and recovery of additives incorporated into a plastic polymer can be also difficult and usually requires the complete dissociation and solvation of the polymer material using hazardous solvents such as xylene at high temperature $\left(>140^{\circ} \mathrm{C}\right)$. With our protocol, heating LLINs with heptane at $85^{\circ} \mathrm{C}$ for 45 min was sufficient to recover insecticides (permethrin, alpha-cypermethrin and pyriproxyfen) from the polyethylene fibers by swelling of the polymer without dissolving the fibre. Similarly, iso-octane has been tested previously as a universal solvent for pyrethroid extraction from polyester and polyethylene nets without dissolving fibre ${ }^{16}$. However, the extraction was reliant on large sample size and lacked an internal standard ${ }^{16}$, thus prone to variability in insecticide quantification due to solvent volatility. In contrast, our method doesn't preclude the internal standard (DCP) recommended in the original CIPAC protocol ${ }^{15}$, resulting in a more robust and reproducible method for the quantitative analysis of the active ingredients from LLINs (Fig. 5-7).

The new method facilitates the analysis of insecticides by enabling multiple net samples to be processed in parallel using standard low volume tubes and multiwall dry blocks for solvent evaporation (Fig. 2). 
Coupled with the higher-sensitivity of HPLC and shorter run times, this greatly speeds up the processing and data collection to analyze LLIN insecticide content. In our hands, one operator can run up to 40 LLINs in triplicate per HPLC run. Moreover, the stability of the insecticides has not been altered during the extraction process as indicated from heat stability data (Table 4) which should result in no alteration of their biological activity. Collectively this qualifies our protocol to be used for quality control purposes to measure pyriproxyfen and pyrethroid content incorporated in LLINs as demonstrated by the use of the method in field trials in Burkina Faso and Benin that tested the efficacy of Olyset ${ }^{\circledR}$ Duo LLIN (Ngufor et al., 2016; Toe et al., 2019). Here, the optimised method has been further refined and evaluated for linearity, specificity, accuracy and precision and found suitable for insecticide quantification from various types of LLINs that incorporate pyriproxyfen, permethrin and alpha-cypermethrin. These include the commercially available Olyset ${ }^{\circledR}$ Net that contains permethrin and has been used extensively for malaria control operations in Africa and Royal Guard ${ }^{\circledR}$ Net a new LLIN that contains a mixture of alpha-cypermethrin and pyriproxyfen and whose use is likely to escalate in future.

The optimised method, which allows the scale-up of insecticide extraction from LLINs offers a relatively simple and cost effective means of performing analytical checks for QCA purposes that would be accessible for most laboratories. Moreover, we anticipate that our method will be valid for other prequalified approved ITNs by PQT-VC (Supplementary data 1) contain pyrethroid insecticides and is the subject of future research.

\section{Declarations}

Funding: The Innovative Vector Control Consortium who are supported by the Bill and Melinda Gates Foundation, under grant number OPP1148615 and iiCON “Delivering Integrated Solutions for Human Infections" who are supported by UKRI SIPF Wave 1, under Ref number 107136 funded this work.

Acknowledgments: Sumitomo Chemical Ltd and Avec Net project are acknowledged for the provision of LLINs. We would also like to thank Professor Hilary Ranson for reading the manuscript and her valuable comments.

Author Contributions: Conceptualization, H.M.I.; Methodology, H.M.I., K.J.W., C.T.W., F.O.O. ; Resources, D.M. and J.L. Data Curation and Analysis, H.M.I. and M.J.I.P.; Writing - Original Draft Preparation, H.M.I. ; Writing - Review \& Editing, H.M.I., K.W., C.W., J.L. and M.J.I.P Supervision, H.M.I. All authors read and approved the final manuscript.

\section{References}

1. WHO. World malaria report. Geneva. Switzerland, (2018).

2. Bhatt, S. et al. The effect of malaria control on Plasmodium falciparum in Africa between 2000 and 2015. Nature 526, 207-211, doi:10.1038/nature15535 (2015).

3. WHO. World malaria report. Geneva. Switzerland, (2020). 
4. Zaim, M., Aitio, A. \& Nakashima, N. Safety of pyrethroid-treated mosquito nets. Med Vet Entomo/ 14, 1-5, doi:10.1046/j.1365-2915.2000.00211.x (2000).

5. Strode, C., Donegan, S., Garner, P., Enayati, A. A. \& Hemingway, J. The impact of pyrethroid resistance on the efficacy of insecticide-treated bed nets against African anopheline mosquitoes: systematic review and meta-analysis. PLoS Med 11, e1001619, doi:10.1371/journal.pmed.1001619 (2014).

6. Ranson, H. et al. Pyrethroid resistance in African anopheline mosquitoes: what are the implications for malaria control? Trends Parasitol 27, 91-98, doi:10.1016/j.pt.2010.08.004 (2011).

7. Ngufor, C. et al. Efficacy of the Olyset Duo net against insecticide-resistant mosquito vectors of malaria. Sci Transl Med 8, 356ra121, doi:10.1126/scitransImed.aad3270 (2016).

8. Mosha, J. F. et al. Protocol for a four parallel-arm, single-blind, cluster-randomised trial to assess the effectiveness of three types of dual active ingredient treated nets compared to pyrethroid-only longlasting insecticidal nets to prevent malaria transmitted by pyrethroid insecticide-resistant vector mosquitoes in Tanzania. BMJ Open 11, e046664, doi:10.1136/bmjopen-2020-046664 (2021).

9. Denholm, I. \& Rowland, M. W. Tactics for managing pesticide resistance in arthropods: theory and practice. Annu Rev Entomo/ 37, 91-112, doi:10.1146/annurev.en.37.010192.000515 (1992).

10. Ngufor, C. et al. Olyset Duo(R) (a pyriproxyfen and permethrin mixture net): an experimental hut trial against pyrethroid resistant Anopheles gambiae and Culex quinquefasciatus in Southern Benin. PLoS One 9, e93603, doi:10.1371/journal.pone.0093603 (2014).

11. Toe, K. H. et al. Assessing the impact of the addition of pyriproxyfen on the durability of permethrintreated bed nets in Burkina Faso: a compound-randomized controlled trial. Malar J 18, 383, doi:10.1186/s12936-019-3018-1 (2019).

12. Ngufor, C., Agbevo, A., Fagbohoun, J., Fongnikin, A. \& Rowland, M. Efficacy of Royal Guard, a new alpha-cypermethrin and pyriproxyfen treated mosquito net, against pyrethroid-resistant malaria vectors. Sci Rep 10, 12227, doi:10.1038/s41598-020-69109-5 (2020).

13. WHO. Guidelines for laboratory and field-testing of long-lasting insecticidal nets World Health Organization (2013).

14. CIPAC. Permethrin long lasting insecticidal net 331/LN/M. Collaborative International Pesticides Analytical Council Handbook M, 158 (2007).

15. CIPAC. Pyriproxyfen long lasting insecticidal net *715/LN/M/. Collaborative International Pesticides Analytical Council Handbook 0, 143 (2014).

16. Jenkins, D. W. et al. Development and validation of a 'universal' HPLC method for pyrethroid quantification in long-lasting insecticidal mosquito nets for malaria control and prevention. Trop Med Int Health 18, 2-11, doi:10.1111/tmi.12011 (2013).

17. Welton, T. Solvents and sustainable chemistry. Proc Math Phys Eng Sci 471, 20150502, doi:10.1098/rspa.2015.0502 (2015).

18. Liu, W., Qin, S. \& Gan, J. Chiral stability of synthetic pyrethroid insecticides. J Agric Food Chem 53, 3814-3820, doi:10.1021/jf048425i (2005). 
19. Harburguer, L. V. et al. Thermal behaviour and biological activity against Aedes aegypti (Diptera: Culicidae) of permethrin and pyriproxyfen in a smoke-generating formulation. Pest Manag Sci 65, 1208-1214, doi:10.1002/ps.1811 (2009).

20. Clarke, C. J., Tu, W. C., Levers, O., Brohl, A. \& Hallett, J. P. Green and Sustainable Solvents in Chemical Processes. Chem Rev 118, 747-800, doi:10.1021/acs.chemrev.7b00571 (2018).

21. Prat, D., Hayler, J. \& Wells, A. A survey of solvent selection guides. Green Chemistry 16, 4546-4551, doi:10.1039/C4GC01149J (2014).

\section{Figures}<smiles>CC1(C)[C](C(=O)OCc2cccc(Oc3ccccc3)c2)[C@@H]1C=C(Cl)Cl</smiles><smiles>CC1(C)[C@H](C=C(Cl)Cl)[C@@H]1C(=O)OCc1cccc(Oc2ccccc2)c1</smiles>

Permethrin<smiles>CC1(C)[C@@H](C(=O)O[C@](C)(N)c2cccc(Oc3ccccc3)c2)[C@@H]1C=C(Cl)Cl</smiles>

(1R, cis)

(Alpha-S)<smiles>CC1(C)C(=CC(Cl)Cl)C1C(=O)O[C@H](C#N)c1cccc(Oc2ccccc2)c1</smiles>

Alpha-cypermethrin<smiles>CC(=Cc1ccccn1)COc1ccc(Oc2ccccc2)cc1</smiles><smiles>CC(COc1ccc(Oc2ccccc2)cc1)Cc1ccccn1</smiles>

Pyriproxyfen

\section{Figure 1}

Chemical structure of permethrin, alpha-cypermethrin and pyriproxyfen insecticides ( ${ }^{*}$ : chiral centres). 


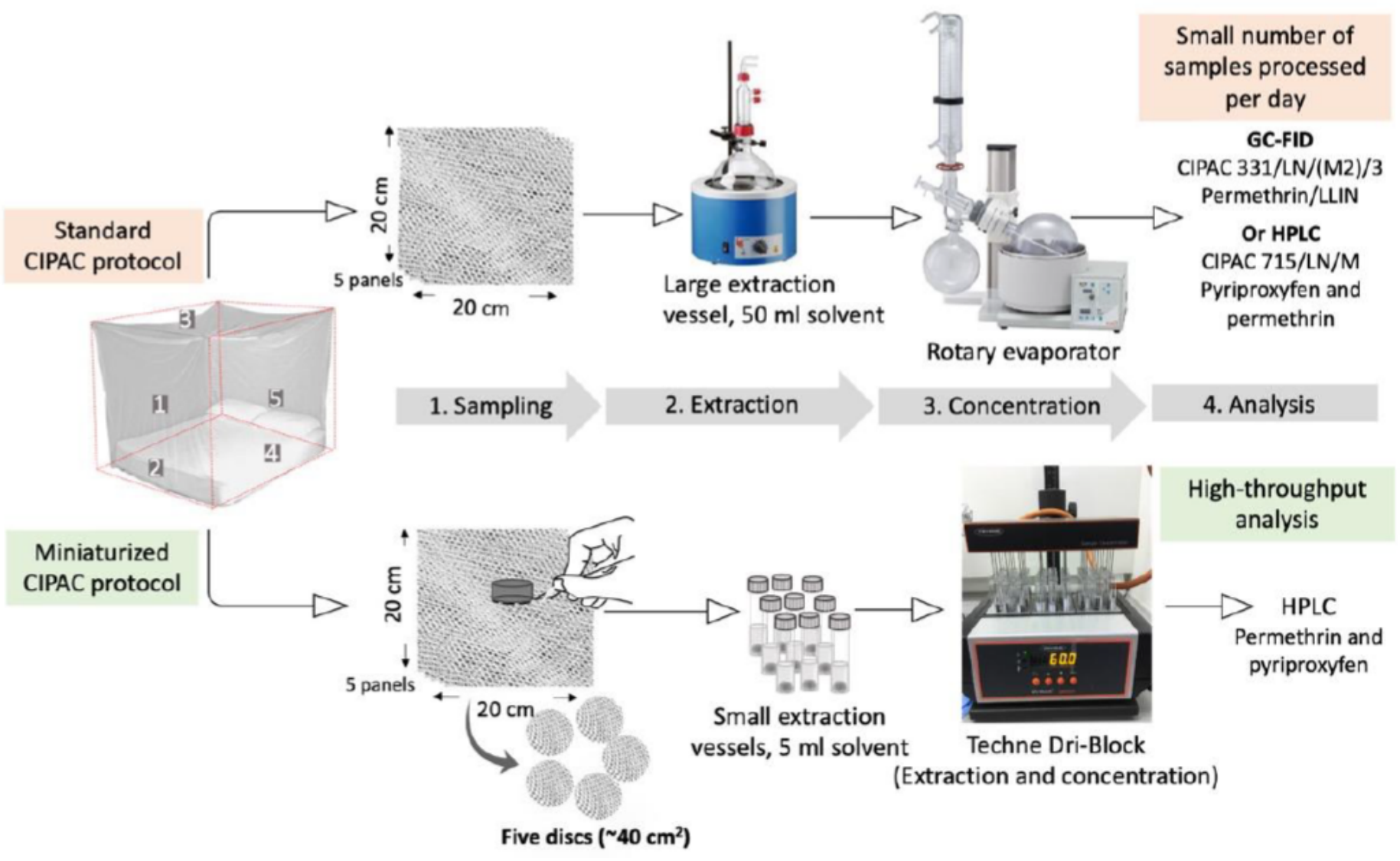

Figure 2

Comparison of standard CIPAC method with a miniaturised protocol for determining insecticide content incorporated in long-lasting bed nets (LLINs). The sample size has been reduced from $400 \mathrm{~cm}^{2}(2 \mathrm{~g})$ to $\sim 40 \mathrm{~cm}^{2}(0.2 \mathrm{~g})$ to enable a small volume of extraction solution (5 vs 50 used in the standard CIPAC methods) for permethrin ${ }^{14}$ and pyriproxyfen ${ }^{15}$ respectively. 

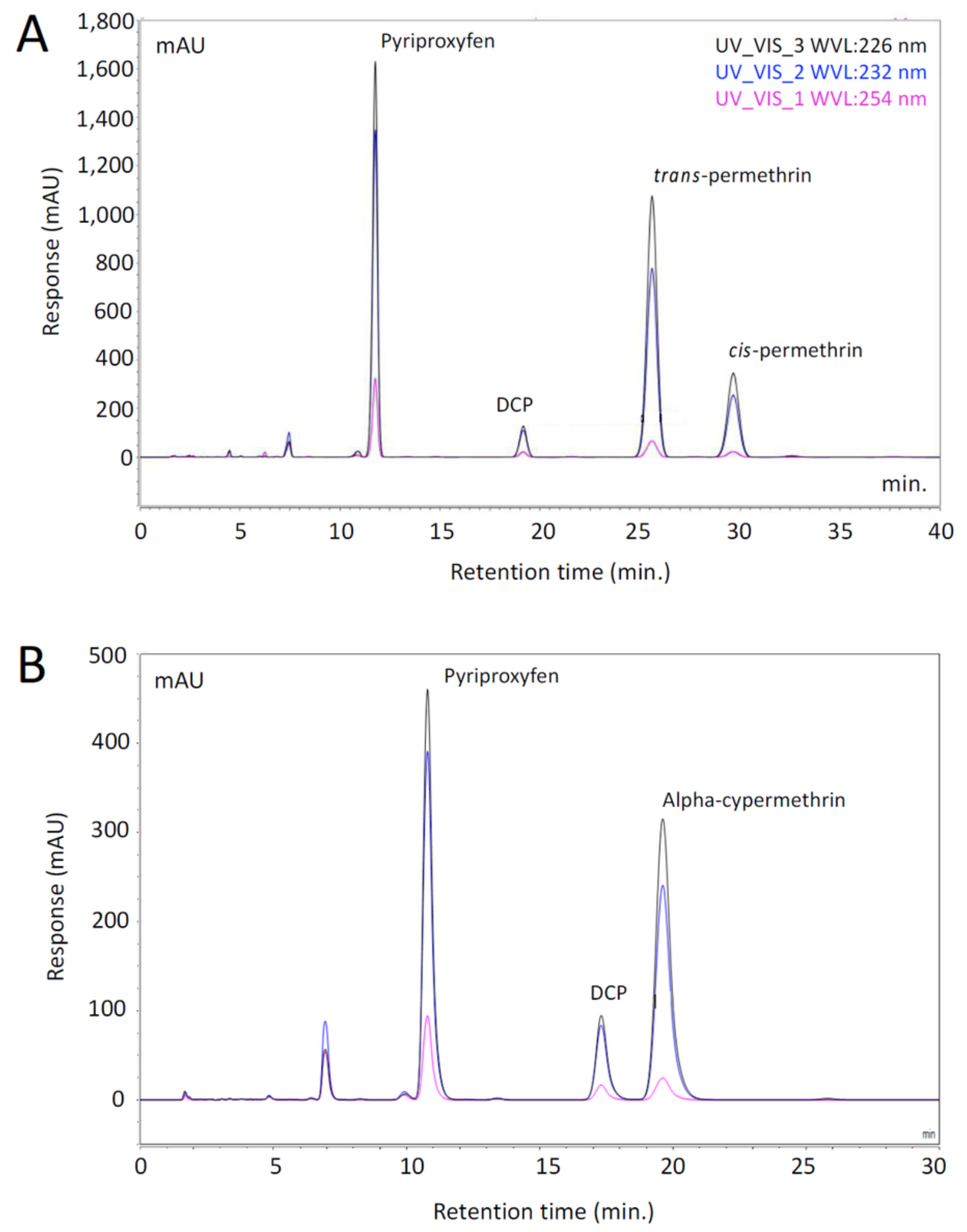

\section{Figure 3}

HPLC chromatogram for pyriproxyfen and pyrethroids extracted from Olyset ${ }^{\circledR}$ Duo and Royal Guard ${ }^{\circledR}$ LLINs with reference to internal standard 'dicyclohexyl phthalate (DCP). (A) Olyset ${ }^{\circledR}$ Duo active ingredients, pyriproxyfen and trans-permethrin and cis permethrin, measured by HPLC-diode array detector (DAD) at three-wavelength 226 (black), 232 (blue) and 254 (purple) nm in LLIN extraction 
solution. (B) Royal Guard ${ }^{\circledR}$ active ingredients; pyriproxyfen, and alpha-cypermethrin, were detected at the same three-wavelength in the sample solution following LLIN extraction.
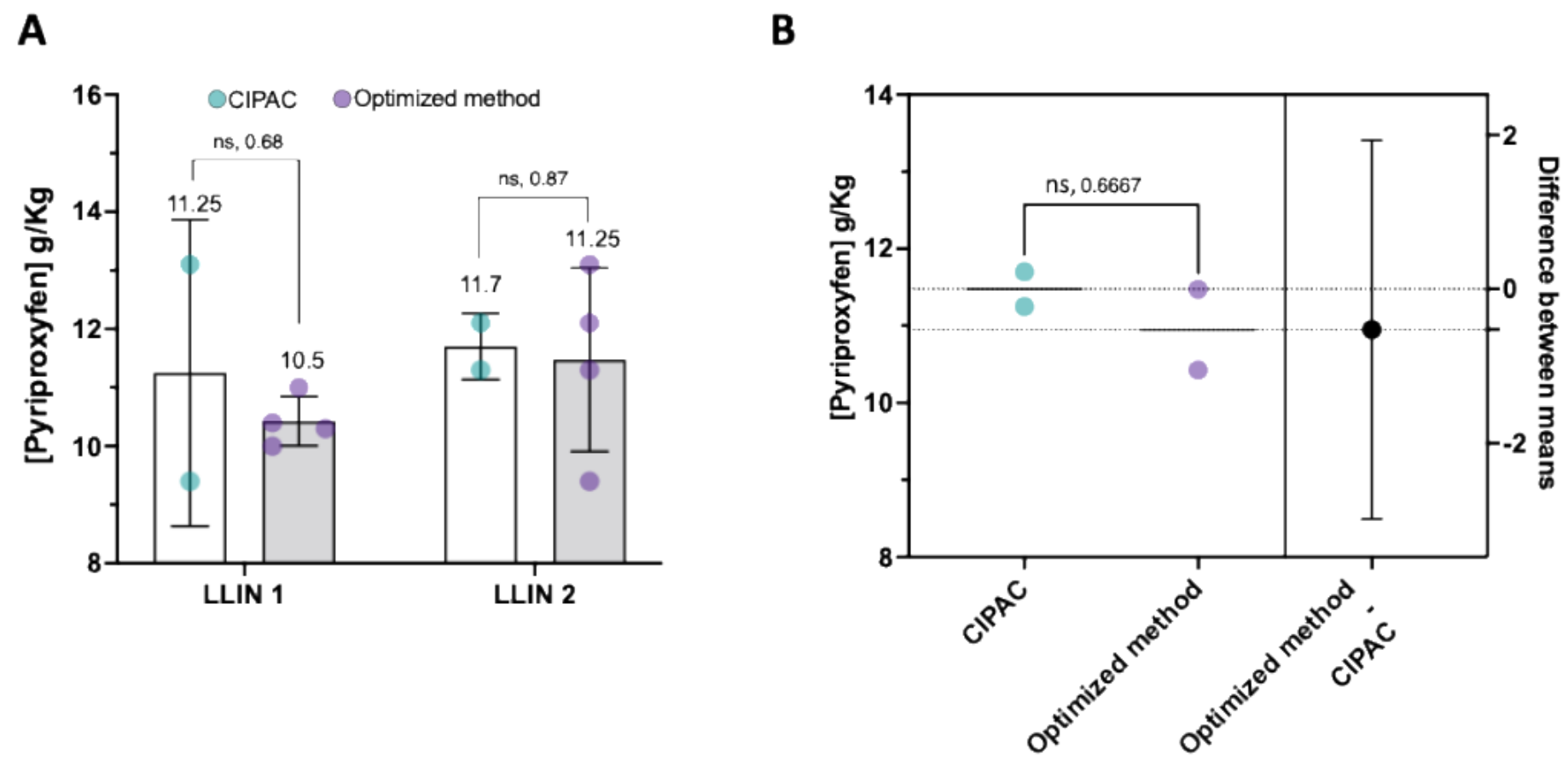

\section{Figure 4}

Comparison of pyriproxyfen content in prototype pyriproxyfen-treated LLINs by standard CIPAC and optimized method. (A) Quantity of pyriproxyfen recovered from pyriproxyfen-LLINs by standard CIPAC protocol vs optimized method. Multiple comparison tests were used to compare the significance of variation between the pyriproxyfen content estimated by the two methods for each LLIN. (B) The magnitude of difference between the optimized method and established CIPAC protocol $(0.5250 \pm$ $0.5712)$ with $95 \% \mathrm{Cl}(-2.983$ to 1.933$)$. An unpaired $t$-test was used to calculate the significant difference between the two methods at the $p$-value of 0.67 . ns; no significance. 
A

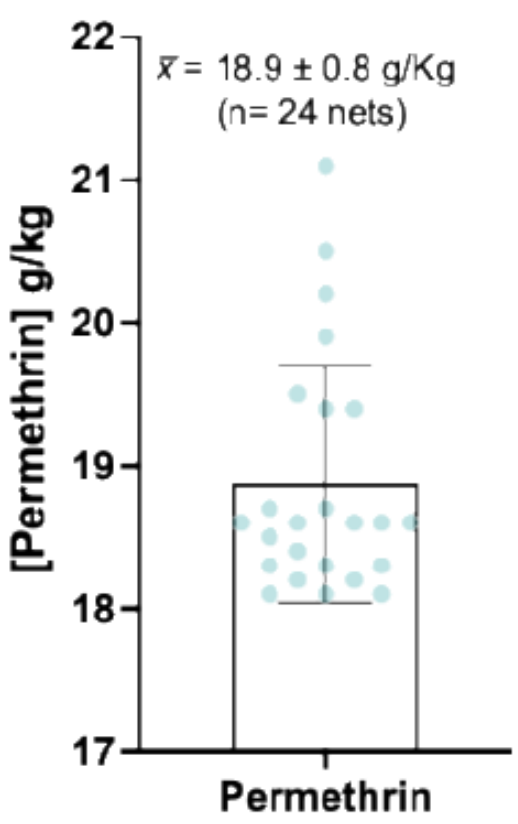

B

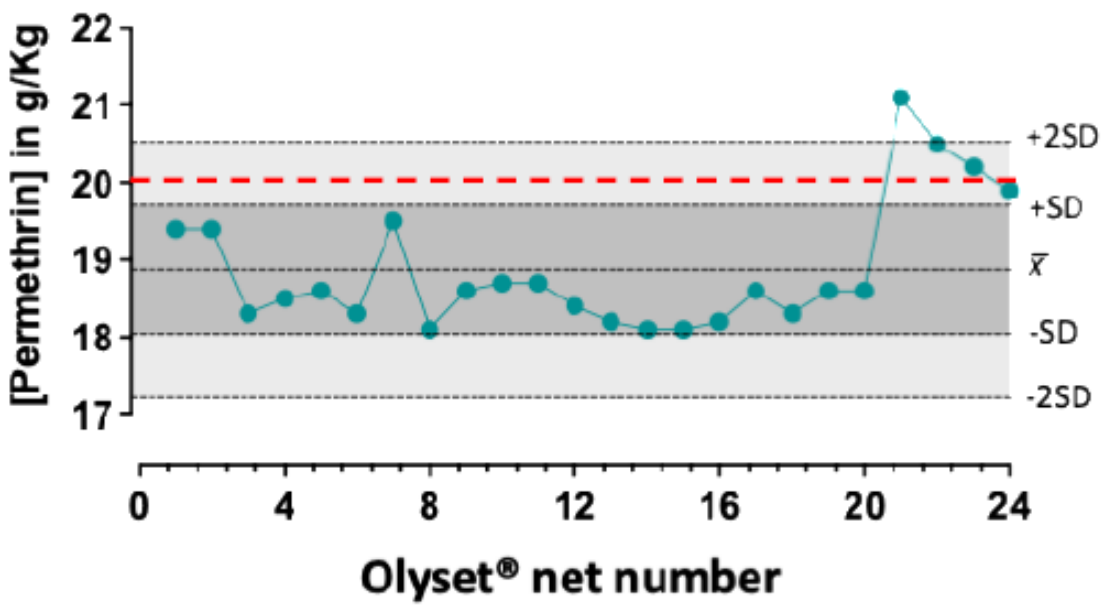

Figure 5

Analysis of total permethrin content in Olyset ${ }^{\circledR}$ net. (A) Permethrin \pm standard deviation (SD) for 24 nets analyzed by the optimized method. (B) Levy-Jenning's chart for pyriproxyfen content in 24 LLINs was analyzed in triplicate (72 samples in total) by the optimized method. An average $(\overline{\mathrm{x}})$ of $18.9 \pm 0.8 \mathrm{~g}$ permethrin $/ \mathrm{kg}(\mathrm{w} / \mathrm{w})$ determined for Olyse ${ }^{\circledR} \mathrm{Net}(\mathrm{n}=24)$ in reference to the target concentration of 20 $\mathrm{g} / \mathrm{kg}$ as declared by the manufacturer and indicated as a dotted red line on the graph. 
A

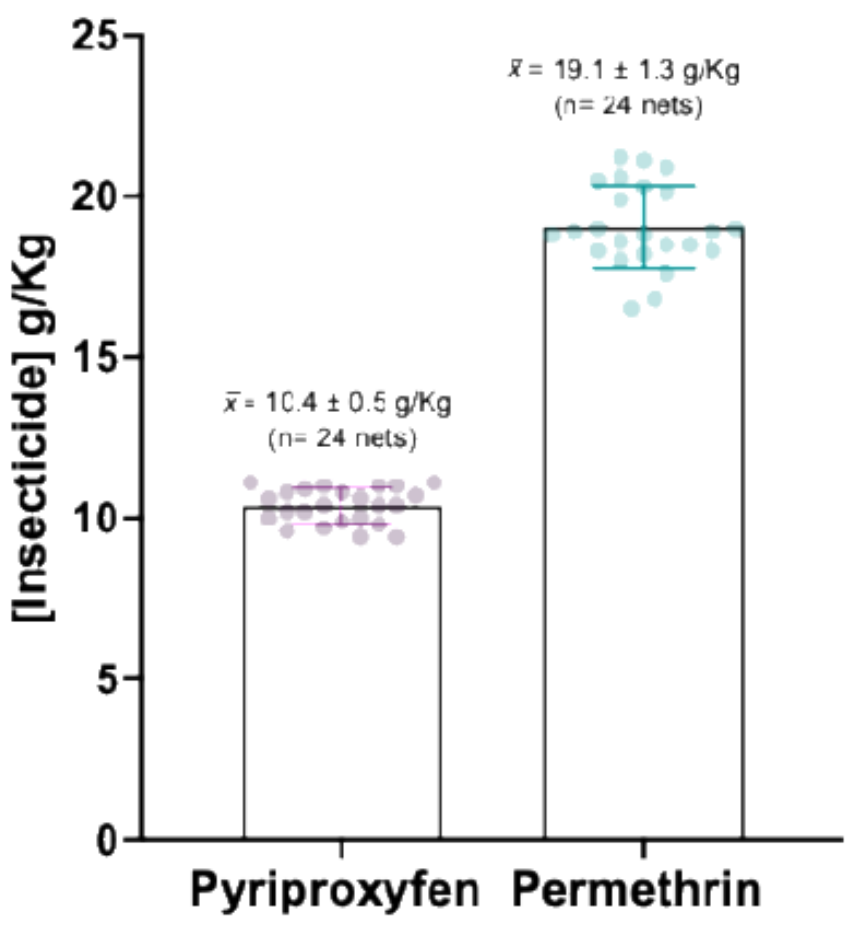

B


Figure 6

Analysis of total pyriproxyfen and permethrin content in Olyset ${ }^{\circledR}$ Duo LLIN. (A) The optimised method analysed the average content of pyriproxyfen and permethrin \pm standard deviation (SD) for 24 Olyset ${ }^{\circledR}$ Duo. (B) Levy-Jenning's chart for the 24 nets analyzed in triplicates ( $n=72$ samples) by the optimized method. Pyriproxyfen (top chart) and permethrin (bottom chart) scored an average ( $\bar{x})$ of $10 \pm 0.5$ and $19.1 \pm 1.3 \mathrm{~g} / \mathrm{kg}$, respectively. Reference concentrations for both active ingredients declared by the manufacture are denoted as red dotted lines on the charts. 


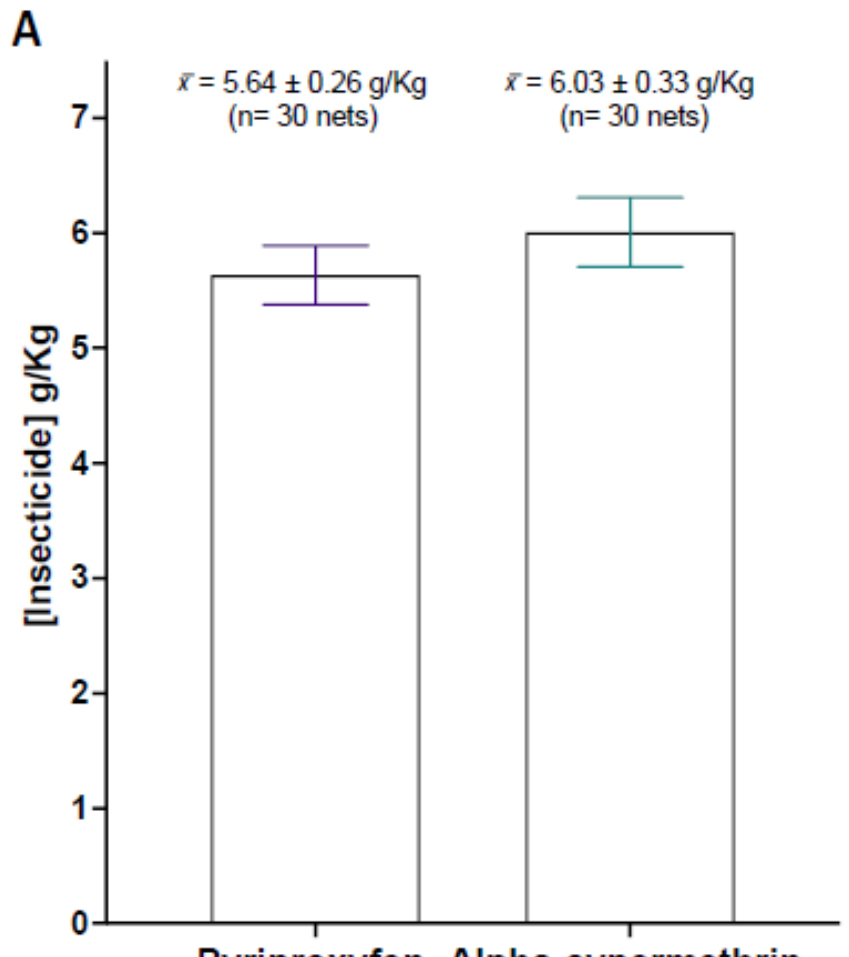

Pyriproxyfen Alpha-cypermethrin
B
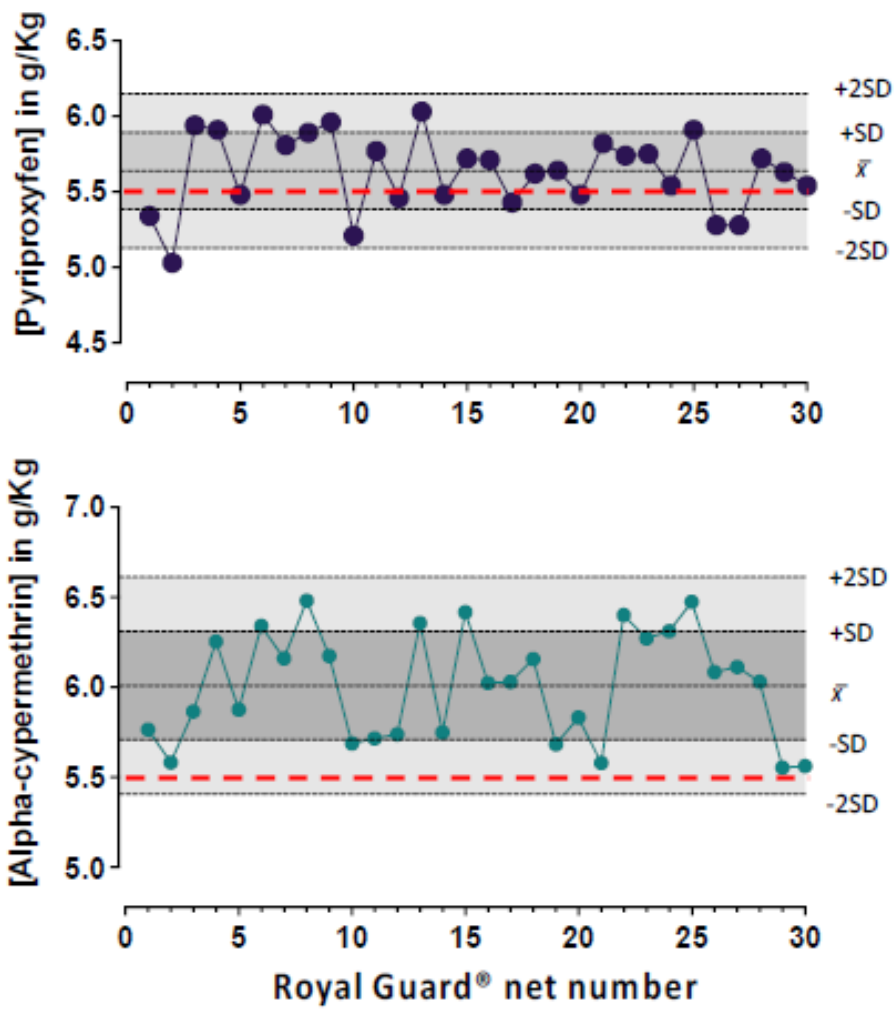

Figure 7

Analysis of total pyriproxyfen and alpha-cypermethrin content in Royal Guard ${ }^{\circledR}$ LLIN. (A) The average content of pyriproxyfen and alpha-cypermethrin \pm standard deviation (S.D.) for 30 Royal Guard ${ }^{\circledR}$ nets. (B) Levy-Jenning's charts for the 30 nets were analyzed by the optimized method. Pyriproxyfen (top chart) and alpha-cypermethrin (bottom chart) scored an average $(\overline{\mathrm{x}})$ of $5.64 \pm 0.26$ and $6.03 \pm 0.33 \mathrm{~g} / \mathrm{kg}$, respectively. Reference concentrations for both active ingredients declared by the manufacture are denoted as red dotted lines on the charts.

\section{Supplementary Files}

This is a list of supplementary files associated with this preprint. Click to download.

- Supplimintarymaterials.docx

- Supplementarydata1.xls 